\title{
Micellar and Polymer Catalysis in the Kinetics of Oxidation of L-lysine by Permanganate Ion in Perchloric Acid Medium
}

\author{
Mohammed Hassan ${ }^{\mathrm{a}, c, *(\mathbb{D})}$, Mohammad Al-Dhoun ${ }^{\mathrm{b}}$, Yazan Batineh ${ }^{\mathrm{b}}$, Ahmad A. Najjar ${ }^{\mathrm{b}}$, \\ Adnan Dahadha ${ }^{a}$ and Qutaiba A. Ibrahim ${ }^{b}$ \\ ${ }^{a}$ Department of Basic Sciences and Mathematics, Faculty of Science, Philadelphia University, Amman, Jordan. \\ ${ }^{b}$ Faculty of Pharmacy, Philadelphia University, Amman, Jordan. \\ ${ }^{c}$ Department of Chemistry, Faculty of Science, Ibb University, Ibb, Yemen. \\ Received 5 July 2020, revised 23 October 2020, accepted 28 October 2020.
}

\begin{abstract}
Kinetics of oxidation of L-lysine by permanganate ion in a perchloric acid medium was investigated to explore the order of the reaction with respect to oxidant and substrate and to study the catalytic behaviour of sodium lauryl sulphate (SLS) and polyethylene glycol (PEG). The reaction was found to be first-order with respect to the oxidant and the substrate and zero-order with respect to hydrogen ion. Changes in the sodium sulphate concentration produce a non-significant variation in the rate of the reaction. SLS and PEG were found to catalyze the reaction. Surfactant catalysis was modelled by Piszkiewicz's cooperativity model, while polymer catalysis was explained with the help of the Benesi-Hildebrand equation. The temperature dependence of the rate of the reaction was elucidated, and activation parameters were obtained. Interestingly, the reaction was found to possess positive activation entropy indicating the dissociative nature of the transition state and outer-sphere electron transfer mechanism. A mechanism of the reaction that is supported by the experimental findings was suggested.
\end{abstract}

KEYWORDS

L-lysine, permanganate ion, micellar catalysis, polymer catalysis, outer sphere electron transfer mechanism.

\section{Introduction}

Oxidation of lysine in its biological form is important and is related to nuclear sclerosis of the lens of the eye and other disorders. ${ }^{1,2}$ Due to lysine and other amino acids' functional role, the oxidation kinetics, with different metal ion oxidants, are of special interest and have undergone extensive investigation. ${ }^{3-6}$ One of the metal ion oxidants of interest is the permanganate heptavalent ion. It is a powerful oxidizing agent and has been used extensively in the oxidation of organic substrates. Its advantages over other oxidants include ease of tracking by visible spectroscopy and titrimetric methods, good applicability of Lambert-Beer's law, and its reduced form is environment friendly, especially with reactions in an acidic medium. The types of active oxidizing species, reaction intermediates, and products depend on the $\mathrm{pH}$ of the reaction solution and the type of substrate. ${ }^{7}$

Surfactants are amphiphilic species, having both hydrophilic and hydrophobic moieties, and at a certain concentration (critical micelle concentration, $\mathrm{CMC}$ ), tend to aggregate in well-defined shaped chemical entities called micelles. Micellar aggregates in aqueous solutions resemble biological membranes. Thus the study of their effect on electron transfer reactions would be of extreme importance in understanding various biological, therapeutic, and energy-storing processes. ${ }^{8}$ One of the interesting properties of surfactants that have received considerable attention over the last two decades is their catalytic behaviour for reactions in aqueous solutions. The catalytic action of surfactants starts at a concentration below their CMC and may level off at a concentration beyond CMC. However, surfactants may also inhibit chemical reactions. ${ }^{9,10}$ The catalytic or inhibition action of surfactants depends on how substrate

* To whom correspondence should be addressed. E-mail: m-hassan@ibbuniv.edu.ye interacts with surfactants aggregates either in premicellar or post-micellar concentrations. Hence, the final influence would depend on the type of surfactant and reaction substrates.

Some polymers were known to catalyze reactions. ${ }^{11,12}$ The catalytic mechanism may be due to the solubilizing of the reactants into the phase in which another reactant exists. Alternatively, the polymer may interact with one or more reactants and concentrate them on the polymer surface and/or palisades. Eventually, the reaction takes place in a different phase, the polymer phase, in which reactants can have more chances to meet and finally speeding up the reaction. Polymeric materials that are soluble in an aqueous solution are of particular interest since they must have many hydrophilic moieties, leading to a more significant role in the catalysis reaction. One of these polymeric materials is polyethylene glycol (PEG), which has attracted the attention of several researchers studying catalytic behaviour. ${ }^{13,14}$ In this study, we investigated the kinetics of the permanganate ion oxidation of L-lysine in a perchloric acid medium. The reaction was already studied in an alkaline medium, where the product was reported to contain decarboxylated residue. In this residue, the $\alpha$-carbon acquires an aldehydic group to eliminate the $\alpha$-amino group in the form of ammonia or ammonium ion. ${ }^{15,16}$ It is known that amino acids are zwitterions, and they are $\mathrm{pH}$ dependent. So, it would be interesting to identify the product of the reaction in the acidic medium and suggest a plausible mechanism. In addition, exploring micellar and polymer catalysis will increase the knowledge about the catalytic behaviour of these interesting chemical species.

\section{Experimental}

\subsection{Materials}

All materials used were of AR or LR grade and were used without further purification. Stock solutions of $0.01 \mathrm{~mol} \mathrm{dm}^{-3}$ 
L-lysine ( $\geq 97 \%$, SAFC, USA), $0.001 \mathrm{~mol} \mathrm{dm}^{-3}$ potassium permanganate (99\%, AZ Chem), $2.0 \mathrm{~mol} \mathrm{dm}^{-3}$ perchloric acid (70 \%, Merck, Germany), and $0.5 \mathrm{~mol} \mathrm{dm^{-3 }}$ sodium sulphate (99\%, Merck, Germany) were prepared using the appropriate weighted amounts of each substance and distilled water. The potassium permanganate stock solution was protected from light with aluminium foil and was used within 48 hours. Solutions of $0.05 \mathrm{~mol} \mathrm{dm}^{-3}$ of sodium lauryl sulphate, SLS (99\%, AZ Chem), and $0.01 \mathrm{~mol} \mathrm{dm}^{-3}$ polyethylene glycol PEG (PEG-400, Schariau, Spain) were prepared just before use.

\subsection{Kinetic Measurements}

All experimental runs were carried out under pseudo-firstorder conditions, in which the concentration of L-lysine was at least one order of magnitude higher than that of permanganate ion. In a principal experimental run, $5 \mathrm{~mL}$ of L-lysine, $5 \mathrm{~mL}$ of perchloric acid, $3 \mathrm{~mL}$ of sodium sulphate and $4 \mathrm{~mL}$ of distilled water were added to the reaction vessel kept in a thermostated water bath along with a tube contained $3 \mathrm{~mL}$ of potassium permanganate. The sodium sulphate was added to the reaction mixture to keep the ionic strength constant during the experiments. The water bath was a PRECISION GP20 water bath manufactured by Thermo Scientific (USA). It controls the temperature digitally to the required $\pm 0.5{ }^{\circ} \mathrm{C}$. After thermal equilibrium was reached, the potassium permanganate was added to the reaction vessel, mixed thoroughly and transferred to a quartz cell of the UV-VIS spectrometer (AE-S90-2D, A and E Lab, UK). The absorbance at $525 \mathrm{~nm}$ was monitored at different time intervals. In subsequent runs, the effects of changing the concentrations of various components, including SLS and PEG, on the rate of the reaction were tested by varying the volume of each component on the account of the volume of water to ensure keeping the overall volume of the mixture constant at $20 \mathrm{~mL}$. The precision of measurements was ensured by repeating each experiment three times and calculating the average values. These values were used for further calculations and analysis.

\subsection{Stoichiometry and Product Identification}

The reaction stoichiometry was determined by preparing three reaction mixtures, in which the concentration of potassium permanganate was in excess over that of L-lysine. The solutions were kept in the dark at ambient temperature for 36 hours until the reaction was completed. The unreacted quantity of potassium permanganate in each solution was determined by titrating with standard $0.01 \mathrm{~mol} \mathrm{dm} \mathrm{dm}^{-3}$ sodium thiosulfate solution. The results indicated that 4 moles of permanganate ion react with 5 moles of L-lysine, hence the balanced equation could be written as follows:

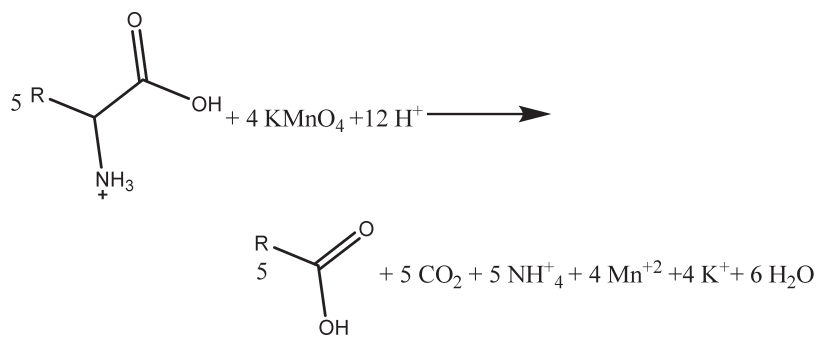

To confirm the expectation that an aldehyde derivative of decarboxylated residue formed during the reaction, a saturated solution of 2,4-dinitropheylhydrazine in $2 \mathrm{~mol} \mathrm{dm}^{-3}$ hydrochloric acid was added to the three solutions mentioned above. Surprisingly, the derivative 2,4-dinitropheylhydrazone did not form, indicating that the aldehydic carbonyl group was not present in the product mixture. So the product is most probably carboxylic acid of the residue, as indicated in Equation 1, which agrees with stoichiometry results. However, the reaction stoichiometry determination required an excess of potassium permanganate in the reaction mixture. Under these conditions, even when the aldehyde formed, it would have been oxidized by the excess potassium permanganate to carboxylic acid. ${ }^{17}$

\section{Results and Discussion}

\subsection{UV-Visible Spectra}

The spectra of permanganate ion and L-lysine are presented in the inset in Fig. 1. It depicts the usual bands for the permanganate ion, as reported in the literature at 525, 340 and $200 \mathrm{~nm}$. An important note is that the band at $200 \mathrm{~nm}$ coincides and overlaps the intense absorption of L-lysine at the same point. Both bands are attributed to the allowed $\pi$ to $\pi^{*}$ electron transition. L-lysine also shows a very weak band that appeared as a small shoulder centred close to $275 \mathrm{~nm}$ due to the forbidden $\mathrm{n}$ to $\pi^{*}$ transition. Spectra of the reaction mixture at different time intervals are shown in Fig. 1. Though the two bands of permanganate ion at 340 and $525 \mathrm{~nm}$ fades quickly, the one close to $200 \mathrm{~nm}$ shows a slow decrease. This slow decrease is due to a counteract absorption of the product at the same position, which could be considered evidence for the presence of the carbonyl group within the structure of the product. There is no observable shift in the position of the three bands of permanganate ion, which indicates that the electron transfer reaction between the oxidant and substrate occurs through the outer-sphere mechanism rather than the inner sphere mechanism that involves the formation of a covalent oxidant-substrate complex. ${ }^{18}$

\subsection{Reaction Order with respect to Oxidant}

The natural logarithm plots of absorbance of the reaction mixture against time for some experimental runs are shown in Fig. 2. Except for a short curvature segment at the beginning of the reaction, the relation is linear during the remaining course of the reaction, which indicates the applicability of pseudo-firstorder kinetics. The observed rate constants $\left(\mathrm{k}_{\mathrm{obs}}\right)$ at pseudofirst-order conditions were calculated by fitting curves to straight lines using the least square method with regression coefficients of $R^{2} \geq 20.99$. These values at various conditions are given in Table 1 . The varying initial concentration of permanganate ion with keeping all other conditions fixed did not significantly alter $\mathrm{k}_{\mathrm{obs}}$ value, which indicates a first-order reaction with

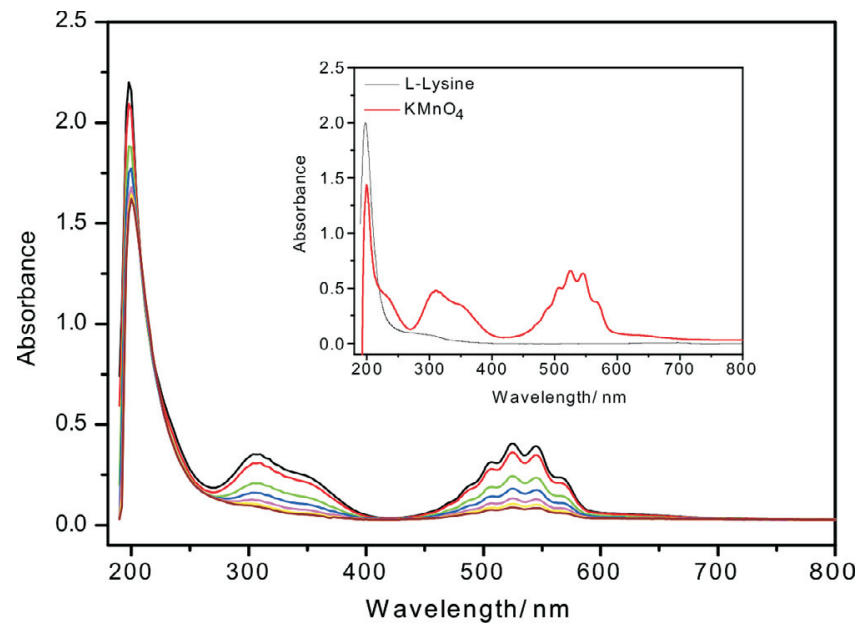

Figure 1 Absorption spectra of the reaction mixture at different time intervals. Inset: The spectra of aqueous solutions of permanganate ion and L-lysine at $298 \mathrm{~K}$. 
respect to the oxidant. In many cases, oxidation of organic substrates, ${ }^{19,20}$ including some amino acids, ${ }^{21}$ in the presence of a permanganate ion occurs according to an autocatalytic pathway. However, this pathway was not observed in this work.

\subsection{Reaction Order with respect to L-lysine}

The increase of the L-lysine concentration resulted in a linear increase in the $\mathrm{k}_{\mathrm{obs}}$ value. The relation, shown in Fig. 2, was fitted successfully to a straight line that passed through the origin $\left(R^{2} \approx 0.98\right)$. This result indicated that the order with respect to L-lysine is unity. The reaction rate constant $\left(\mathrm{k}_{2}\right)$ was found to be $0.23 \mathrm{~mol}^{-1} \mathrm{dm}^{3} \mathrm{~s}^{-1}$ at $298 \mathrm{~K}$. The double logarithm plot of $\mathrm{k}_{\mathrm{obs}}$ and L-lysine concentrations was successfully fitted to a straight line (inset in Fig. 3) with a slope close to unity, which is another evidence for the first-order kinetics of L-lysine.

\subsection{Effect of Acid and Salt Concentrations}

Several experimental runs were performed in which the concentration of perchloric acid or sodium sulfate was varied. All other pseudo-order conditions were fixed at $298 \mathrm{~K}$. The values of $\mathrm{k}_{\mathrm{obs}}$ were calculated in the same manner mentioned above and tabulated in Table 1 . These values show no significant changes with the varying perchloric acid concentration, which indicates that the order with respect to hydrogen ion is zero. The absence of the hydrogen ion in the reaction mixture was found to affect the reaction product. The colour turned to a yellowishbrown, indicating the formation of manganese dioxide instead of the divalent manganese. However, the hydrogen ion is necessary to proceed to the targeted product, even though it is not

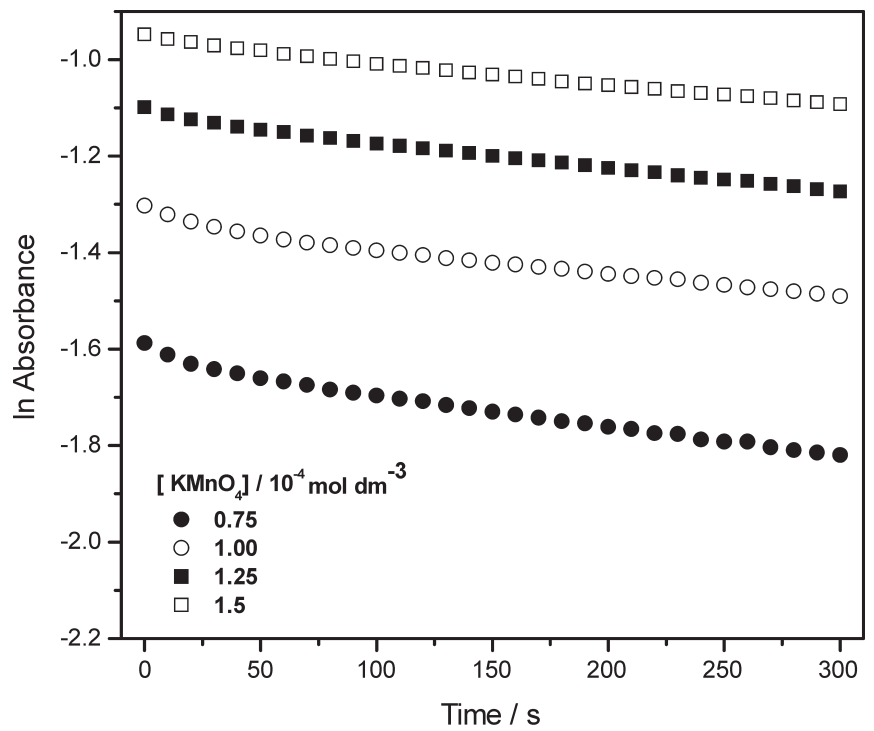

Figure 2 Pseudo-first-order reaction time curves at varying concentration of permanganate ion and other fixed condition: [L-lysine] $=2.5 \times$ $10^{-3} \mathrm{~mol} \mathrm{dm}^{-3},\left[\mathrm{HClO}_{4}\right]=0.5 \mathrm{~mol} \mathrm{dm}^{-3},\left[\mathrm{Na}_{2} \mathrm{SO}_{4}\right]=0.075 \mathrm{~mol} \mathrm{dm}^{-3}$, and at 298 K.

involved in the rate-determining step. Therefore, its reaction order is zero. Its role in this reaction is important in later stages and is involved in the disproportional transformation of $\mathrm{Mn}(\mathrm{V})$ to $\mathrm{Mn}(\mathrm{VII})$ and $\mathrm{Mn}(\mathrm{II})$, as is shown in the reaction mechanism.

Values of $\mathrm{k}_{\mathrm{obs}}$ are also independent of sodium sulphate concen-

Table 1 The values of $k_{\text {obs }}$ at various pseudo-first-order conditions at $298 \mathrm{~K}$.

\begin{tabular}{|c|c|c|c|c|c|c|}
\hline $\begin{array}{c}10^{3}[\text { L-lysine }] \\
\mathrm{mol} \mathrm{dm}^{-3}\end{array}$ & $\begin{array}{c}10^{4}\left[\mathrm{KMnO}_{4}\right] \\
\mathrm{mol} \mathrm{dm}^{-3}\end{array}$ & $\begin{array}{l}{\left[\mathrm{HClO}_{4}\right]} \\
\mathrm{mol} \mathrm{dm}\end{array}$ & $\begin{array}{l}{\left[\mathrm{Na}_{2} \mathrm{SO}_{4}\right]} \\
\mathrm{mol} \mathrm{dm}^{-3}\end{array}$ & $\begin{array}{l}10^{4}[\mathrm{SLS}] \\
\mathrm{mol} \mathrm{dm}^{-3}\end{array}$ & $\begin{array}{l}10^{4}[\mathrm{PEG}] \\
\mathrm{mol} \mathrm{dm}^{-3}\end{array}$ & $10^{4} \mathrm{k}_{\mathrm{obs}} \mathrm{s}^{-1}$ \\
\hline 2.5 & 1.50 & 0.5 & 0.075 & - & - & 4.9 \\
\hline 2.5 & 0.75 & 0.5 & 0.075 & - & - & 5.2 \\
\hline 2.5 & 1.00 & 0.5 & 0.075 & - & - & 5.0 \\
\hline 2.5 & 1.25 & 0.5 & 0.075 & - & - & 5.4 \\
\hline 3.0 & 1.50 & 0.5 & 0.075 & - & - & 5.0 \\
\hline 3.5 & 1.50 & 0.5 & 0.075 & - & - & 6.1 \\
\hline 4.0 & 1.50 & 0.5 & 0.075 & - & - & 7.3 \\
\hline 4.5 & 1.50 & 0.5 & 0.075 & - & - & 8.5 \\
\hline 2.5 & 1.50 & 0.5 & 0.100 & - & - & 5.0 \\
\hline 2.5 & 1.50 & 0.5 & 0.125 & - & - & 5.2 \\
\hline 2.5 & 1.50 & 0.5 & 0.150 & - & - & 4.9 \\
\hline 2.5 & 1.50 & 0.6 & 0.075 & - & - & 5.0 \\
\hline 2.5 & 1.50 & 0.7 & 0.075 & - & - & 5.2 \\
\hline 2.5 & 1.50 & 0.8 & 0.075 & - & - & 5.0 \\
\hline 2.5 & 1.50 & 0.5 & 0.075 & 1.25 & - & 5.5 \\
\hline 2.5 & 1.50 & 0.5 & 0.075 & 5.00 & - & 5.8 \\
\hline 2.5 & 1.50 & 0.5 & 0.075 & 12.5 & - & 6.2 \\
\hline 2.5 & 1.50 & 0.5 & 0.075 & 50.0 & - & 7.7 \\
\hline 2.5 & 1.50 & 0.5 & 0.075 & 80.0 & - & 10.4 \\
\hline 2.5 & 1.50 & 0.5 & 0.075 & 100 & - & 9.6 \\
\hline 2.5 & 1.50 & 0.5 & 0.075 & - & 2.5 & 6.3 \\
\hline 2.5 & 1.50 & 0.5 & 0.075 & - & 7.5 & 7.4 \\
\hline 2.5 & 1.50 & 0.5 & 0.075 & - & 15 & 8.9 \\
\hline 2.5 & 1.50 & 0.5 & 0.075 & - & 25 & 11.6 \\
\hline 2.5 & 1.50 & 0.5 & 0.075 & - & 30 & 11.8 \\
\hline
\end{tabular}




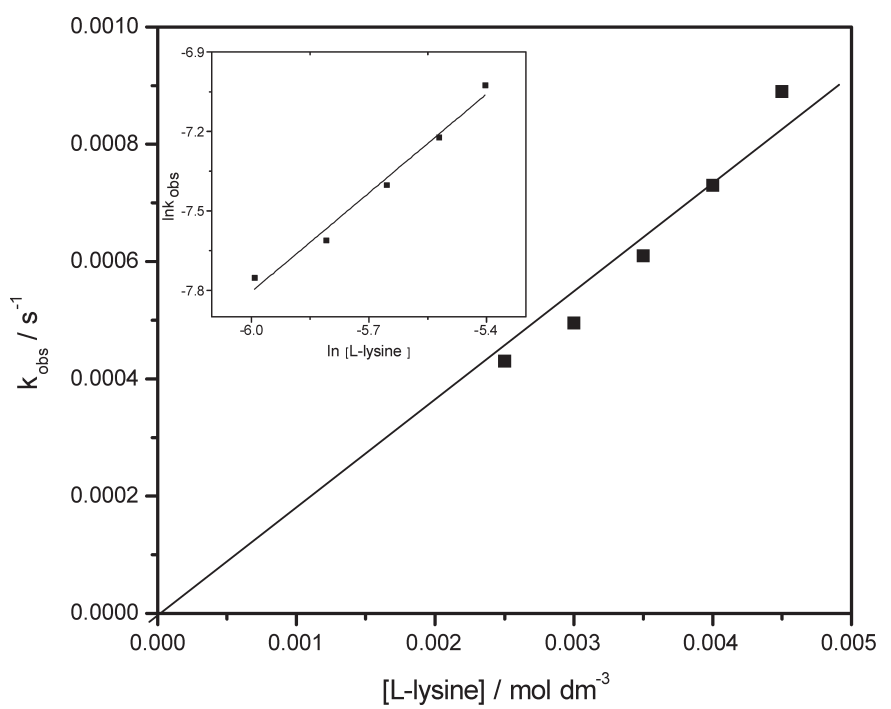

Figure $3 \mathrm{k}_{\mathrm{obs}}$ versus molar concentration of L-lysine at the following conditions: $\left[\mathrm{KMnO}_{4}\right]=1.5 \times 10^{-4} \mathrm{~mol} \mathrm{dm}^{-3},\left[\mathrm{HClO}_{4}\right]=0.5 \mathrm{~mol} \mathrm{dm}{ }^{-3}$, $\left[\mathrm{Na}_{2} \mathrm{SO}_{4}\right]=0.075 \mathrm{~mol} \mathrm{dm}^{-3}$ at $298 \mathrm{~K}$. The inset shows the relation between $\ln \mathrm{k}_{\mathrm{obs}}$ and $\ln$ [L-lysine] at the same conditions.

tration. The null salt effect indicates that either both reactants are neutral or hold opposite charges. ${ }^{22}$ The predominant L-lysine form in the acidic medium is expected to be the protonated L-lysine $\left(\mathrm{H}_{2} \mathrm{~A}^{+}\right)$, which carries a net positive charge. Because of that, the active species of the oxidant would be $\mathrm{MnO}_{4}{ }^{-}$rather than $\mathrm{HMnO}_{4}$. Although $\mathrm{HMnO}_{4}$ was reported to be the active species of permanganate ion in an acidic medium. ${ }^{23}$

\subsection{Surfactant and Polymer Catalysis}

Variation of the $\mathrm{k}_{\mathrm{obs}}$ value with change in SLS and PEG concentrations is depicted in Fig. 4, A and B, respectively. The value of $\mathrm{k}_{\mathrm{obs}}$ steadily increased with increasing SLS concentration up to some point at which a sudden rise occurred, which was followed by almost levelling off. The $\mathrm{k}_{\text {obs }}$ value was found to level off at an SLS concentration of $7.8 \times 10^{-3} \mathrm{~mol} \mathrm{dm}^{-3}$, which is close to the literature reported CMC of $8.0 \times 10^{-3} \mathrm{~mol} \mathrm{dm}^{-3} .{ }^{24}$ Therefore, it is usually called Kinetic CMC. The maximum $\mathrm{k}_{\mathrm{obs}}$ value obtained $\left(10.4 \times 10^{-4} \mathrm{~s}^{-1}\right)$ was about two times that in the absence of SLS. The catalytic efficiency is much lower than that reported for the SLS catalyzed oxidation of glutamic acid with hexavalent chromium in an acidic medium, in which the $\mathrm{k}_{\mathrm{obs}}$ value was enhanced about ten times. ${ }^{25,26}$

The active oxidant of hexavalent chromium was the protonated species. This species is subjected to electric attraction to the negative head group of SLS. It keeps the substrate close to the core of the surfactants. Hence, the micellar catalysis is the sum of two factors: the oxidant's attraction interaction with the head group and solubilization of substrate in the core. In this work, the expected active oxidant species, as mentioned in the preceded section, is the negatively charged $\mathrm{MnO}_{4}^{-}$. In this case, the solubilizing effect of the substrate will be the only factor that contributes to micellar catalytic efficiency.

Further evidence for this hypothesis could be concluded from the magnitude of the initial absorbance measured at experimental runs containing varying SLS concentration and fixed amounts of other components. It was observed that the initial absorbance of the reaction mixture was not affected by the initial SLS concentration and remained almost constant. This absorbance suggests that no strong interaction might occur between the permanganate ion and the SLS, and the interaction that had caused the catalytic effect involved L-lysine and SLS.

Several models were postulated to explain surfactant catalytic

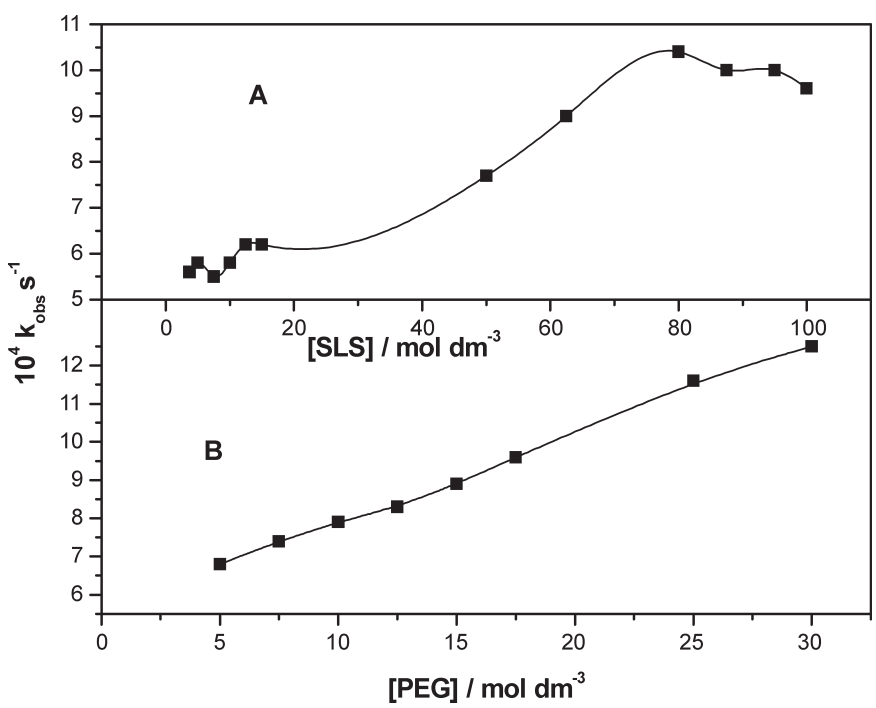

Figure $4 \mathrm{k}_{\mathrm{obs}}$ versus molar concentration of SLS (A), and PEG (B) at the following conditions: [L-lysine] $=2.5 \times 10^{-3} \mathrm{~mol} \mathrm{dm}^{-3},\left[\mathrm{KMnO}_{4}\right]=1.5 \times$ $10^{-4} \mathrm{~mol} \mathrm{dm}^{-3},\left[\mathrm{HClO}_{4}\right]=0.5 \mathrm{~mol} \mathrm{dm}^{-3},\left[\mathrm{Na}_{2} \mathrm{SO}_{4}\right]=0.075 \mathrm{~mol} \mathrm{dm}^{-3}$ at $298 \mathrm{~K}$.

behaviour. However, as the data in this work showed catalysis at low concentrations and levelling off at concentration $\geq \mathrm{CMC}$, it is better discussed in terms of Piszkiewicz's model since this model is best fitted at a concentration lower than CMC. ${ }^{27,28}$ According to this model, catalysis could be achieved by cooperative interaction of one of the reactants with premicellar aggregates, which may be dimers, trimers, tetramers, etc. The reactant induces more micellar aggregation and, at the same time, is being concentrated at the surface of aggregates resulting in a higher rate of reaction. The model leads to the following equation $^{29}$ :

$$
\log \frac{\mathrm{k}_{\psi}-\mathrm{k}_{\mathrm{w}}}{\mathrm{k}_{\mathrm{m}}-\mathrm{k}_{\psi}}=\mathrm{nlog}[\mathrm{D}]-\log \mathrm{K}_{\mathrm{D}}
$$

where $\mathrm{k}_{\mathrm{w}}$ is the rate constant in the absence of surfactant, is the observed rate constant in the micellar aggregates, $\mathrm{k}_{\psi}$ is the observed rate constant in the presence of surfactant, $\mathrm{n}$ is cooperativity index, [D] is surfactant molar concentration and $K_{D}$ is the dissociation constant of the micelle. $n$ and $K_{D}$ can be obtained from the slope and intercept of the straight line that results from plotting $\frac{\mathrm{k}_{\psi}-\mathrm{k}_{\mathrm{w}}}{\mathrm{k}_{\mathrm{m}}-\mathrm{k}_{\psi}}$ versus [D]. Such relation has been graphically represented in Fig. 5A, where it shows satisfactory fitting to a straight line with a slope of 1.40 and intercept of 3.47. Accordingly, the value of cooperativity index (n) is 1.40, larger than unity, indicating a positive cooperative interaction. ${ }^{30}$ The calculated $K_{D}$ was found to be $3.39 \times 10^{-4}$. The value of $n$ was found to lie within the range of those reported for SLS when used with other $\alpha$-amino acids oxidized by chloramine-T; however, the value of $\mathrm{K}_{\mathrm{D}}$ was found to be one order of magnitude higher. ${ }^{31}$

Figure $4 \mathrm{~B}$ shows how the value of $\mathrm{k}_{\mathrm{obs}}$ varies almost linearly with the molar concentration of PEG in the reaction mixture. For the catalytic effect of polymers, it is expected that the effect may level off at some higher polymer concentration that is outside the scope of this work. The level off is expected to occur when the polymer concentration offers excess catalytic sites over that needed for the substrate. It was observed that upon increasing the PEG concentration in subsequent experimental runs, the initial absorbance of the reaction mixture decreased gradually. This decrease indicates that the permanganate ion interacts with PEG. This interaction leads to forming a complex species with 

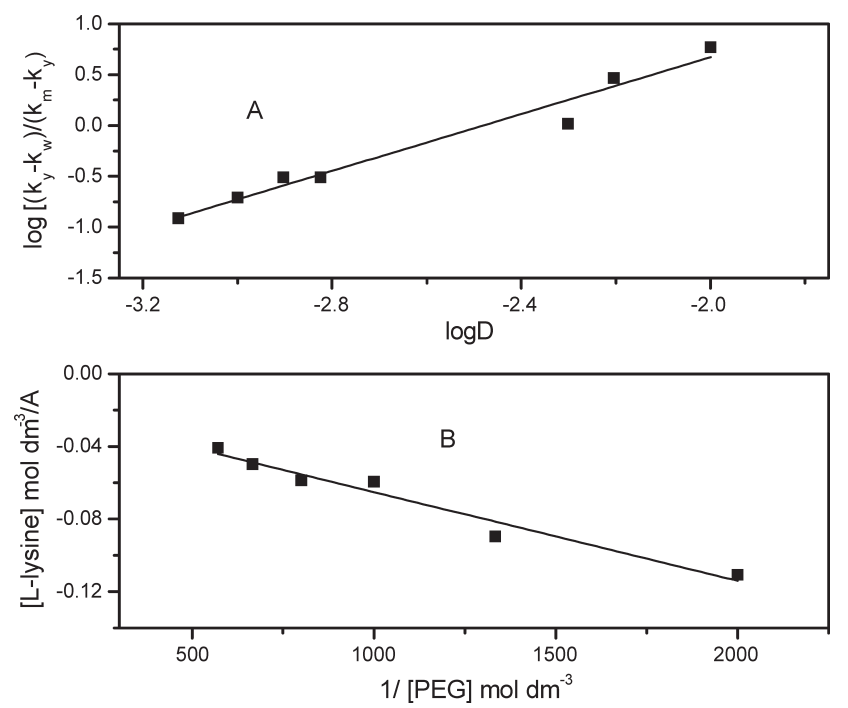

Figure 5 Graphical representation of the application of Piszkiewicz's model on the SLS micellar catalysis (A) and Benesi-Hildebrand model in PEG polymer catalysis (B), of the reaction.

different extinction coefficients, thereby inducing a hypochromic effect. ${ }^{32}$ Experimental runs were carried out in which L-lysine was not added to the mixture to assure the type of interaction between permanganate ion and PEG. The absorbance remained constant with time, which indicates that the interaction of permanganate ion and PEG is non-covalent. The binding constant of the L-lysine-PEG complex can be evaluated by invoking the Benesi-Hildebrand equation that can be adapted to this work in the following form:

$$
\frac{\text { L-lysine }}{\Delta \mathrm{A}}=\frac{1}{\mathrm{~K}[\mathrm{PEG}] \varepsilon}+\frac{1}{\varepsilon}
$$

where $\Delta \mathrm{A}=\mathrm{A}-\mathrm{A}_{\mathrm{KMnO}}$ with $\mathrm{A}$ the initial absorbance of the reaction mixture in the presence of PEG and $\mathrm{A}_{\mathrm{KMnO}}$ the initial absorbance in the absence of PEG, $\varepsilon$ is the extinction coefficient of the L-lysine-PEG complex, and $\mathrm{K}$ is the binding constant. Figure $5 \mathrm{~B}$ shows the plot of $\frac{\mathrm{L} \text {-lysine }}{\mathrm{A}}$ versus $\frac{1}{[\mathrm{PEG}]}$. It was well-fitted to a straight line with a slope of $-4.89 \times 10^{-5}$ and an intercept of -0.0163 . The binding constant $\mathrm{K}$ was calculated by taking the ratio between the intercept and the slope, which was $333.33 \mathrm{M}^{-1}$.

\subsection{Effect of Temperature}

The temperature dependence of $\mathrm{k}_{\mathrm{obs}}$ has been modelled using the Arrhenius equation, which has the following graphically applicable form:

$$
\ln \mathrm{k}_{\mathrm{obs}}=\ln \mathrm{A}-\frac{\mathrm{E}_{\mathrm{a}}}{\mathrm{RT}}
$$

A is called pre-exponential factored, $\mathrm{R}$ is the universal gas constant, $\mathrm{E}_{\mathrm{a}}$ is the reaction's activation energy, and $\mathrm{T}$ is the absolute temperature. Figure $6 \mathrm{~A}$ shows the graphical representation upon application of Equation 4 on the data of this work. The resulting curve has been fitted satisfactorily to a straight line with a slope of -1581.96 and an intercept of -0.01631 . The activation energy of the reaction was calculated from the slope and found to be $13.15 \mathrm{~kJ} \mathrm{~mol}^{-1}$. This value is relatively low and indicates that the reaction rate is less responsive to changes in temperature. For more understanding of the kinetic-thermodynamic correlations in this reaction, the values of other parameters of the transition state, namely activation enthalpy $\Delta \mathrm{H}^{\#}$,
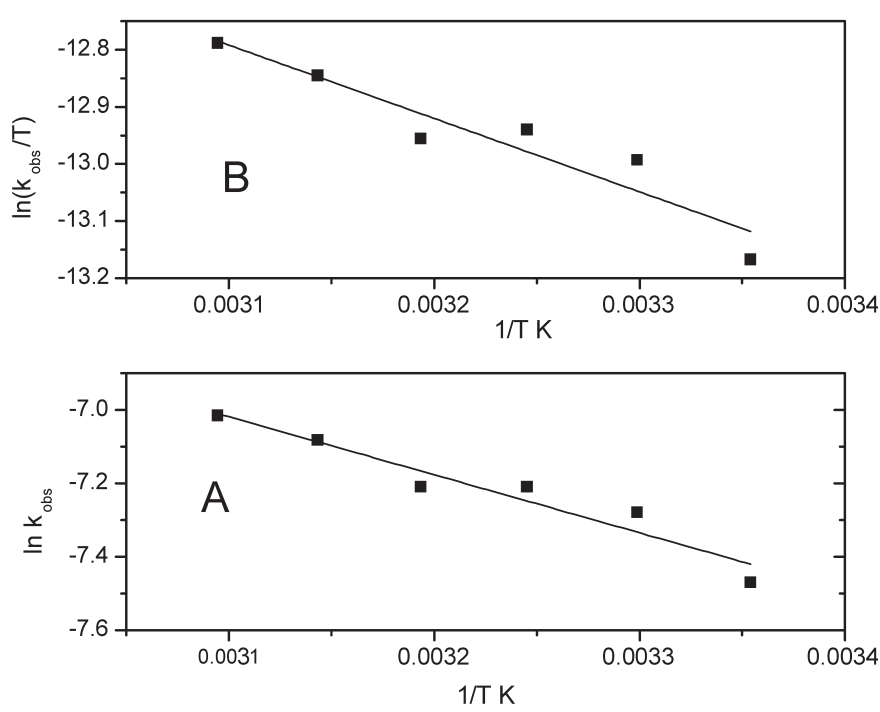

Figure 6 Graphical representation of Arrhenius equation (A), and Eyring equation $(\mathbf{B})$ at the following condition: [L-lysine] $=2.5 \times$ $10^{-3} \mathrm{~mol} \mathrm{dm}^{-3},\left[\mathrm{KMnO}_{4}\right]=1.5 \times 10^{-4} \mathrm{~mol} \mathrm{dm}^{-3},\left[\mathrm{HClO}_{4}\right]=0.5 \mathrm{~mol} \mathrm{dm}{ }^{-3}$, $\left[\mathrm{Na}_{2} \mathrm{SO}_{4}\right]=0.075 \mathrm{~mol} \mathrm{dm}^{-3}$ in the temperature range $298-323 \mathrm{~K}$.

activation entropy $\Delta S^{\#}$, and activation Gibbs energy $\Delta G^{\#}$, have been calculated from the slope and intercept of the graphical plot of the following form of the Eyring equation:

$$
\ln \frac{\mathrm{k}_{\mathrm{obs}}}{\mathrm{T}}=\frac{\Delta \mathrm{H}^{\#}}{\mathrm{RT}}+\ln \frac{\mathrm{K}_{\mathrm{B}} \mathrm{T}}{\mathrm{h}}+\frac{\Delta \mathrm{S}^{\#}}{\mathrm{R}}
$$

where $K_{B}$ is Boltzmann constant. The plot is shown in Fig. $6 B$, where it has been fitted to a straight line. The calculated values of $\Delta \mathrm{H}^{\#}$ and $\Delta \mathrm{S}^{\#}$ are $10.69 \mathrm{~kJ} \mathrm{~mol}^{-1}$ and $171.40 \mathrm{~J} \mathrm{~K}^{-1} \mathrm{~mol}^{-1}$, respectively. The positive activation enthalpy reflects that the formation of the transition state is endothermic. In contrast, the positive and relatively high activation entropy could be evidence of the more complex and asymmetric structure of the transition state compared to those of the reactants due to the dissociative nature of the reaction..$^{33}$ Positive activation entropy is usually considered strong evidence that electron transfer reaction proceeds via an outer-sphere mechanism, which does not involve forming a complex between oxidant and substrate. ${ }^{34}$ Activation Gibbs free energy was calculated using the following equation:

$$
\Delta \mathrm{G}^{\#}=\Delta \mathrm{H}^{\#}-\mathrm{T} \Delta \mathrm{S}^{\#}
$$

$\Delta \mathrm{G}^{\#}$ was found to be $-40.47 \mathrm{~kJ} \mathrm{~mol}^{-1}$. A negative activation Gibbs free energy indicates ease of transition state formation and its relatively high tendency to be converted to the products rather than return to the reactants. So the reaction is spontaneous and of a relatively high rate.

\section{Mechanism}

Depending on the salt effect results (section 3.4), the permanganate ion participates in the slow step of the reaction as $\mathrm{MnO}_{4}^{-}$ while L-lysine in the form of protonated positive form. Besides, spectroscopic results and the positive activation entropy suggest an outer-sphere mechanism for the reaction. The final product, as indicated by the stoichiometry experiments, is the 5-amino pentanoic acid. Hence, the probable mechanism is suggested as in Scheme 1. The reaction rate is independent of hydrogen ion concentration because the active form of the oxidant is $\mathrm{MnO}^{-}{ }_{4} . \mathrm{HMnO}_{4}$ is classified as a strong acid. ${ }^{35}$ Hence even in a strongly acidic medium, permanganate ion exists mainly as $\mathrm{MnO}_{4}^{-}$. 


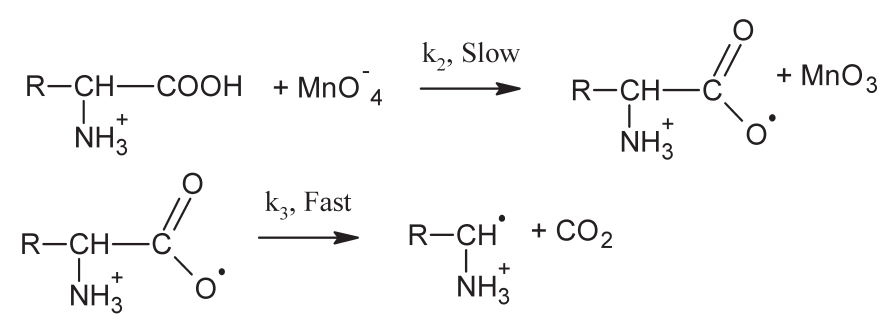

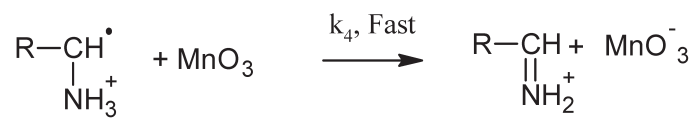

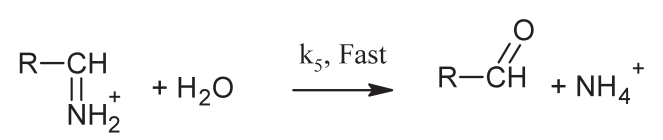

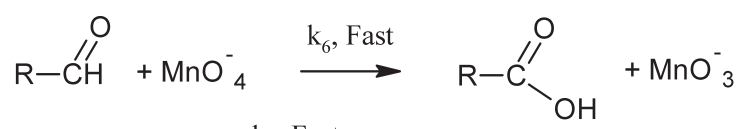

$5 \mathrm{MnO}_{3}^{-}+2 \mathrm{H}^{+} \stackrel{\mathrm{k}_{7}, \text { Fast }}{\longrightarrow} 3 \mathrm{MnO}_{4}^{-}+2 \mathrm{MnO}+\mathrm{H}_{2} \mathrm{O}$

Scheme 1

\section{Conclusion}

The kinetics of L-lysine oxidation by permanganate ion in perchloric acid medium has been investigated under pseudofirst-order conditions. The reaction was found to be first order with respect to each of the oxidant and substrate and zero order with respect to hydrogen ion. The rate of the reaction showed no significant variation with changing the ionic strength. SLS was found to catalyze the reaction in premicellar concentration, and the rate of the reaction reached a maximum value at the CMC concentration of SLS. The micellar catalysis was explained using cooperative Piszkiewicz's model. The reaction rate increased with increasing PEG concentration without level off observed within the PEG concentration range used in this work. Interestingly, the reaction activation entropy was positive, indicating an outer-sphere mechanism. This suggestion is supported by the absence of any shift in the absorption bands of permanganate ion in UV-Vis spectra of the reaction mixture recorded at subsequent time intervals.

\section{Acknowledgement}

M.H. acknowledges the International Institute of Education (IIE), New York, for the SRF fellowship.

\section{${ }^{8}$ ORCID iD}

M. Hassan: (iD) orcid.org/0000-0003-2531-9360

\section{References}

1 X. Fan, J. Zhang and M. Theves, Christopher Strauch, Ina Nemet, Xiaoqin Liuet al., Mechanism of lysine oxidation in human lens crystallins during aging and in diabetes, J. Biol. Chem., 2009, 284(50), 34618-34627.

2 B.S. Berlett and E.R. Stadtman, Protein oxidation in aging, disease, and oxidative stress, J. Biol. Chem., 1997, 272(33), 20313-20316.

3 I. Amelio, F. Cutruzzola, A. Antonov, M. Agostini and G. Melino, Serine and glycine metabolism in cancer, Trends Biochem, Sci,, 2014, 39(4), 191-198.

4 J.F. Perez-Benito, F. Mata-Perez and E. Brillas, Permanganate oxidation of glycine: kinetics, catalytic effects, and mechanisms, Can. J. Chem., 1987, 65, 2329-2334.

5 S. Sheik Mansoor, V. Saleem Malik, K. Aswin, K. Logaiya and A.M Hussain, Kinetics and thermodynamics of oxidation of some thio acids by tripropylammonium fluorochromate in $\mathrm{N}, \mathrm{N}$-dimethyl formamide and acetic acid mixture, J. Saudi Chem. Soc., 2016, 20, S77-S84.

6 S. Mathur, M.B. Yadav and V. Devra, Oxidation of lysine by chromium (VI) in acid perchlorate medium: a kinetic study, Int. J. Chem. Sci., 2015, 13(2), 641-649.

7 A. Fawzy, I.A. Zaafarany, I. Althagafi, J. Alfahemi and M. Morad, Kinetics and mechanism of oxidation of vanillin by permanganate in neutral medium and the effect of different transition metal ion catalysts, Austin Chem. Eng., 2016, 3(1), 1026-1031.

8 M.H. Mondal, Md.A. Ali, A. Pal and B. Saha, A Review on micellar catalyzed oxidation reactions of organic functional groups in aqueous medium using various transition metals, Tenside Surfactants Detergents, 2019, 56(6), 516-525.

9 Z. Zaheer and Rafiuddin, Sub- and post-micellar catalytic and inhibitory effects of cetlytrimethylammonium bromide in the permanganate oxidation of phenylalanine, Colloids Surf. B: Biointerf., 2009, 69, 251-256.

10 M. Hassan, M.D. AlAhmadi and M. Mosaid, Micellar effect on the kinetics of oxidation of methyl blue by $\mathrm{Ce}(\mathrm{IV})$ in sulfuric acid medium, Arabian J. Chem., 2015, 8, 72-77.

11 S. Itsuno, Polymer Catalysts, in Encyclopedia of Polymeric Nanomaterials, (S. Kobayashi and K. Müllen, eds.), Springer-Verlag, Berlin, Heidelberg, 2015.

12 H. Salimi, A. Rahimi and A. Pourjavadi, Applications of polymeric reagents in organic synthesis, Monatsh. Chem., 2007, 138, 363-379.

13 R.S. Mekala, S.K. Balam, J.P. Harinath, R.R. Gajjal and S.R. Cirandur, Polyethylene glycol (PEG-400): an efficient medium for the synthesis of 1,2-disubstituted benzimidazoles, Cogent Chem., 2015, 1, 1049932(1).

14 S. Shylaja, K.C. Rajanna, K. Ramesh, K. Rajendar Reddy and P. Giridhar Reddy, Polyethylene glycols as efficient catalysts for the oxidation of xanthine alkaloids by ceric ammonium nitrate in acetonitrile: a kinetic and mechanistic approach, Adv. Phys. Chem., 2013, Article ID 835610.

15 A.L. Harihar, M.R. Kembhavi and S.T. Nandibewoor, Kinetics of oxidative degradation of $\mathrm{L}(+)$ lysine by alkaline permanganate - a mechanistic approach, Indian J. Chem., (2000), 39, 769-774.

16 B. Mohanty, J. Behera, S. Acharya, P. Mohanty and A.K. Patnik, Metal ion catalyzed oxidation of L-lysine by alkaline permanganate ion - a kinetic and mechanistic approach, Chem. Sci. Trans., 2013, 2(1), 51-60.

17 S. Kothari, V. Sharma, P.K. Sharma and K.K. Banerji, Kinetic study of the oxidation of aliphatic aldehydes by bis(2,2'-bipyridyl) copper(ll) permanganate, Proc. Indian Acad. Sci. - Chem. Sci., 1992, 104(5), 583-589.

18 V. Jagannadham, Electron transfer reactions: a treatise, Am. J. Chem., 2012, 2(2): 57-82.

19 B.H. Asghar and A. Fawzy, Kinetic, Mechanistic, and spectroscopic studies of permanganate oxidation of azinylformamidines in acidic medium, with autocatalytic behavior of manganese(II), J. Saudi Chem. Soc., 2016, 20, 561-569.

20 M.B. Bolattin, S.T. Nandibewoor and S.A. Chimatadar, Conclusive evidence for autocatalytic behaviour of manganese(II) ions in the oxidative degradation of ondansetron by permanganate in aqueous sulfuric acid medium - a kinetic and mechanistic approach, J. Environ. Chem. Eng., 2015, 3(2): 1233-1242.

21 J.F. Perez-Benito, Permanganate oxidation of $\alpha$-amino acids: kinetic correlations for the nonautocatalytic and autocatalytic reaction pathways. J. Phys. Chem. A, 2011, 115, 9876-9885.

22 M.R. Wright, An Introduction to Chemical Kinetics, John Wiley and Sons, England, 2004, p. 274.

23 A. Fawzy, N. El Guesmi, H.M. Ali and M. Abdallah, Oxidation of tryptophan by permanganate ion in acid, neutral and alkaline media: a comparative kinetic and mechanistic study, J. Mater. Environ. Sci., 2018, 9(6), 1645-1655.

24 P. Mukerjee, Critical Micelle Concentrations of Aqueous Surfactant Systems. Department of Commerce, National Bureau of Standards, Washington, D.C., USA, 1971 p. 66.

25 K. Mukherjee and B. Saha, Rate enhancement by micelle encapsulation for oxidation of L-glutamic acid in aqueous media at room temperature, J. Korean Chem. Soc., 2013, 57(4), 425-431.

26 A. Basu, S.Kr. Ghosh, R. Saha, A. Ghosh, T. Ghosh, K. Mukherjee, S.S. Bhattacharyya and B. Saha, Kinetic studies of glutamic acid oxidation 
